\title{
Einsatz der Grundwassermodellierung zur Unterstützung der quantitativen Beweissicherung beim Bau des BOKU- Forschungsgerinnes Nußdorf
}

\author{
Ernest Mayr · Sebastian Handl · Reinhard Perfler
}

Online publiziert: 15. Juli 2019

(C) Der/die Autor(en) 2019

Zusammenfassung Die Universität für Bodenkultur Wien hat ein Forschungsgerinne für wasserbauliche Zwecke am Standort Wien 19 (Nußdorf), Am Brigittenauer Sporn, mit der Entnahme von Wasser aus der Donau bei Stromkm 1933,350 und der Rückgabe in den Donaukanal bei DK-km 0,500 errichtet. Teile dieser Anlagen liegen in der zum Schutz der Grundwasserressourcen ausgewiesenen Schutzzone III/2 des Wasserwerks Nußdorf. Ziel der Bearbeitungen ist es, Auswirkungen des Forschungsgerinnes auf das Grundwasserdargebot $\mathrm{zu}$ untersuchen und zu dokumentieren. Die Validierung der konkreten Einflüsse der Baumaßnahmen auf die quantitativen Grundwasserverhältnisse lt. wasserrechtlichem Bescheid wird mittels zwei Pumpversuchen durchgeführt. Um den Einfluss des Bauwerks auf den Grundwasserhaushalt darstellen zu können, müssen die Einflüsse der Kolmation und der Temperatur auf die Zielgröße der Absenkung eliminiert werden. Dazu wurde ein numerisches Grundwassermodell erstellt. Die berechneten Unterschiede zwischen den Szenarien mit und ohne Berücksichtigung der Querschnittsminderung durch das Bauwerk für die beiden Pumpversuche liegen aufgrund ihrer geringen Ausprägung sowohl beim Wasserstand als auch bei der Zustromrate unter der Genauigkeit der Modellanpassung an die Beobachtungen. Es ist somit nicht von einer Beeinflussung der quantitativen Grundwasserverhält-

DI E. Mayr $(\varangle) \cdot$ DI S. Handl PD DI Dr. R. Perfler

Department

Wasser-Atmosphäre-Umwelt Institut für Siedlungswasserbau, Industriewasserwirtschaft und Gewässerschutz, Universität für Bodenkultur Wien, Muthgasse 18, 1190 Wien, Österreich ernest.mayr@boku.ac.at nisse des Brunnenfelds Nußdorf durch das Forschungsgerinne auszugehen.

\section{Schlüsselwörter}

Grundwassermodellierung · Einfluss eines Bauwerks · Uferfiltration

\section{Use of groundwater modelling to quantitative evidence preservation for BOKU's hydraulic engineering research channel Nußdorf}

Abstract The University of Natural Resources and Life Sciences Vienna has established a research channel for hydraulic engineering purposes at Vienna 19 (Nußdorf), Am Brigittenauer Sporn, with the withdrawal of water from river Danube at flow-km 1933.350 and the return to the Danube Canal at DK-km 0.500. Parts of the respective facilities are located within the protected zone III/2 of the Nußdorf Waterworks dedicated to the protection of groundwater resources. The present article aims to investigate and document effects of the research channel on the groundwater resources. The validation of specific effects of the construction activities on the quantitative groundwater conditions according to official note is carried out by means of two pumping tests. In order to visualize the effect of the channel on the groundwater regime, effects of colmation and temperature on the target parameter well depression have to be eliminated. For this purpose, a numerical groundwater model was compiled to improve the evaluation of the two pumping tests between the scenarios with and without consideration of cross section reduction. The results show that the effects of the channel structure are below the accuracy of the model adaptation to observations due to their low severity in both the water level and the inflow rate. It is therefore assumed that the quantitative flow of groundwater from the
Brunnenfeld Nußdorf is not influenced by the research channel.

Keywords Groundwater modelling · Impact of structure $\cdot$ Riverbank filtration

\section{Hintergrund}

Die Universität für Bodenkultur Wien hat ein Forschungsgerinne für wasserbauliche Zwecke am Standort Wien 19 (Nußdorf), Am Brigittenauer Sporn, mit der Entnahme von Wasser aus der Donau bei Strom-km 1933,350 und der Rückgabe in den Donaukanal bei DK$\mathrm{km} \mathrm{0,500} \mathrm{errichtet.} \mathrm{Teile} \mathrm{dieser} \mathrm{Anlagen}$ liegen in der zum Schutz der Grundwasserressourcen ausgewiesenen Schutzzone III/2 des Wasserwerks Nußdorf. Um eine quantitative und qualitative Beeinträchtigung des Grundwasserkörpers auszuschließen, wurden In der zugehörigen wasserrechtlichen Bewilligung für das Forschungsgerinne auf der sogenannten Schleuseninsel Nußdorf zwischen Donau und Donaukanal entsprechende Auflagen und Bedingungen formuliert.

Der vermutlich mit dem Brunnenfeld Nußdorf verbundene, ca. 11-12 m mächtige und ca. $20.000 \mathrm{~m}^{2}$ große Grundwasserleiter am Brigittenauer Sporn (ident mit der Ausdehnung von Schutzzone III/2) wird für den Bau des Forschungsgerinnes durch eine zweireihige, überschnittene Bohrpfahlwand über nahezu die gesamte Höhe abgetrennt. Durch diese Teilung verringert sich die mit Wasserwerk Nußdorf potenziell kommunizierende Fläche des Grundwasserleiters auf der Schleuseninsel um ca. $80 \%$ bzw. ist mit einer deutlich schlechteren Anbindung derselben $\mathrm{zu}$ rechnen. Zur Beweissicherung der Auswirkung dieser Reduktion auf die quantitativen Grundwasserverhältnisse ist zunächst die Durchführung jeweils eines Pumpversuchs vor Beginn 

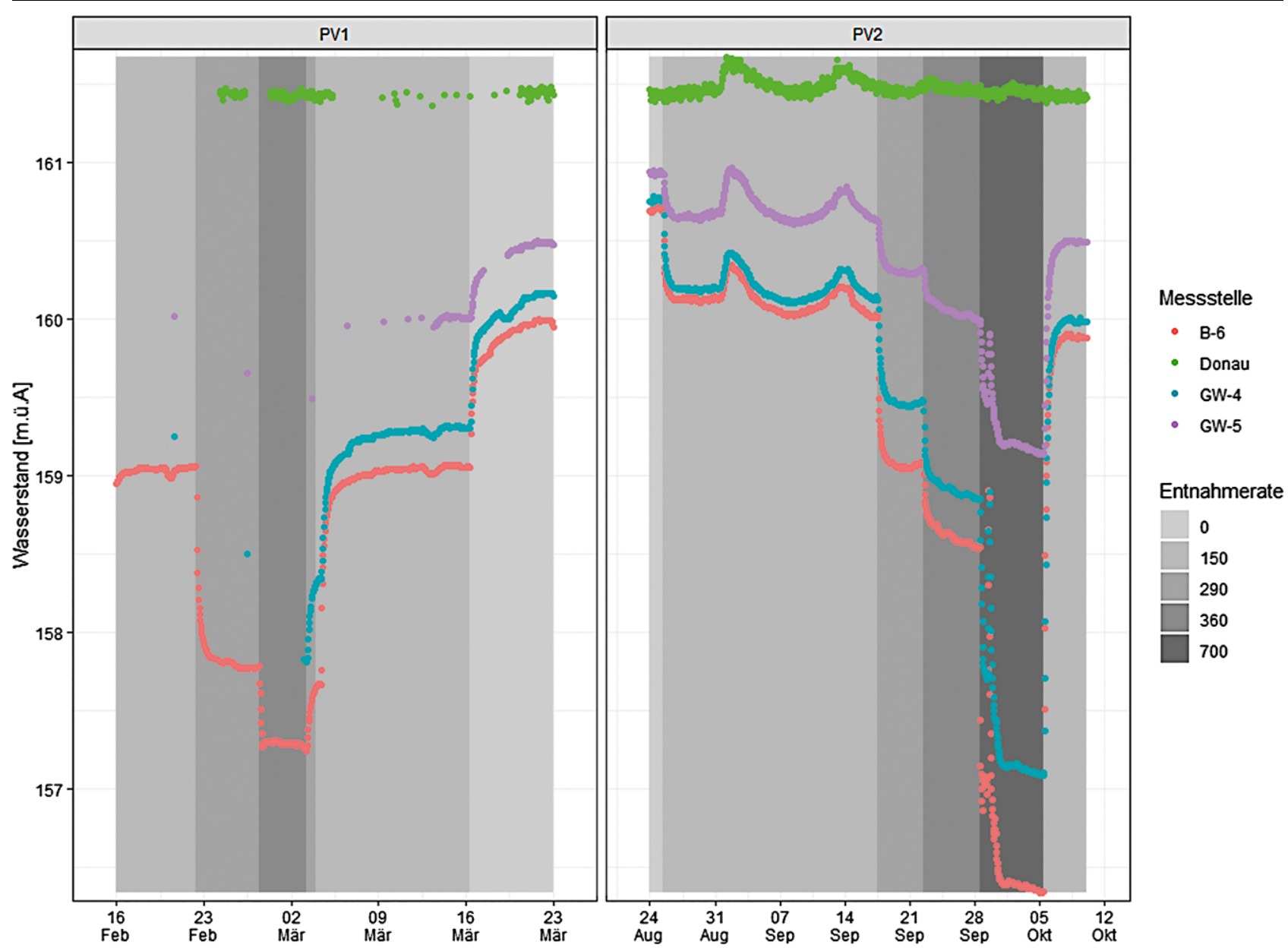

Abb. 1 Wasserstandsverlauf während der Pumpversuche

Tab. 1 Phasen Pumpversuch 1

\begin{tabular}{|l|l|l|l|}
\hline Beginn & Ende & Pumpversuch & Entnahmerate \\
\hline 17.02.2014 & 23.02 .2014 & PV1 & 150 \\
\hline 23.02.2014 & 28.02 .2014 & PV1 & 290 \\
\hline 28.02.2014 & 04.03 .2014 & PV1 & 360 \\
\hline 04.03 .2014 & 05.03 .2014 & PV1 & 290 \\
\hline 05.03 .2014 & 17.03 .2014 & PV1 & 150 \\
\hline 17.03 .2014 & 24.03 .2014 & PV1 & 0 \\
\hline
\end{tabular}

Tab. 2 Phasen Pumpversuch 2

\begin{tabular}{|l|l|l|l|}
\hline Beginn & Ende & Pumpversuch & Entnahmerate \\
\hline 25.08 .2014 & 26.08 .2014 & PV2 & 0 \\
\hline 26.08 .2014 & 18.09 .2014 & PV2 & 150 \\
\hline 18.09 .2014 & 23.09 .2014 & PV2 & 290 \\
\hline 23.09 .2014 & 29.09 .2014 & PV2 & 360 \\
\hline 29.09 .2014 & 06.10 .2014 & PV2 & 700 \\
\hline 06.10 .2014 & 11.10 .2014 & PV2 & 150 \\
\hline
\end{tabular}

und nach Beendigung der Bauarbeiten vorgesehen.

\section{Aufgabenstellung und Zielsetzung}

Ziel der Bearbeitungen ist es, die Ermittlung der Auswirkungen des Forschungsgerinnes auf das Grundwas- serdargebot $\mathrm{zu}$ untersuchen und $\mathrm{zu}$ dokumentieren. Die Validierung der konkreten Einflüsse der Baumaßnahmen auf die quantitativen Grundwasserverhältnisse lt. wasserrechtlichem Bescheid wird mittels zwei Pumpversuchen durchgeführt.

Für die Planung der Pumpversuche kann teilweise auf die Erkenntnisse der jährlich durchgeführten Pumpversuche zur Bestimmung der Kolmation des Gewässerbetts der Donau zurückgegriffen werden. Abhängig von den Erkenntnissen der hydrologischen und hydrogeologischen Beschaffenheit des Untergrunds wurde daraufhin ein Versuchsplan ausgearbeitet und mit dem Betrieb des Wasserwerks Nußdorf abgestimmt. Zur Ausführung gelangen insgesamt zwei Pumpversuche, einer vor Beginn und einer nach Beendigung der Arbeiten im Schutzgebiet. 


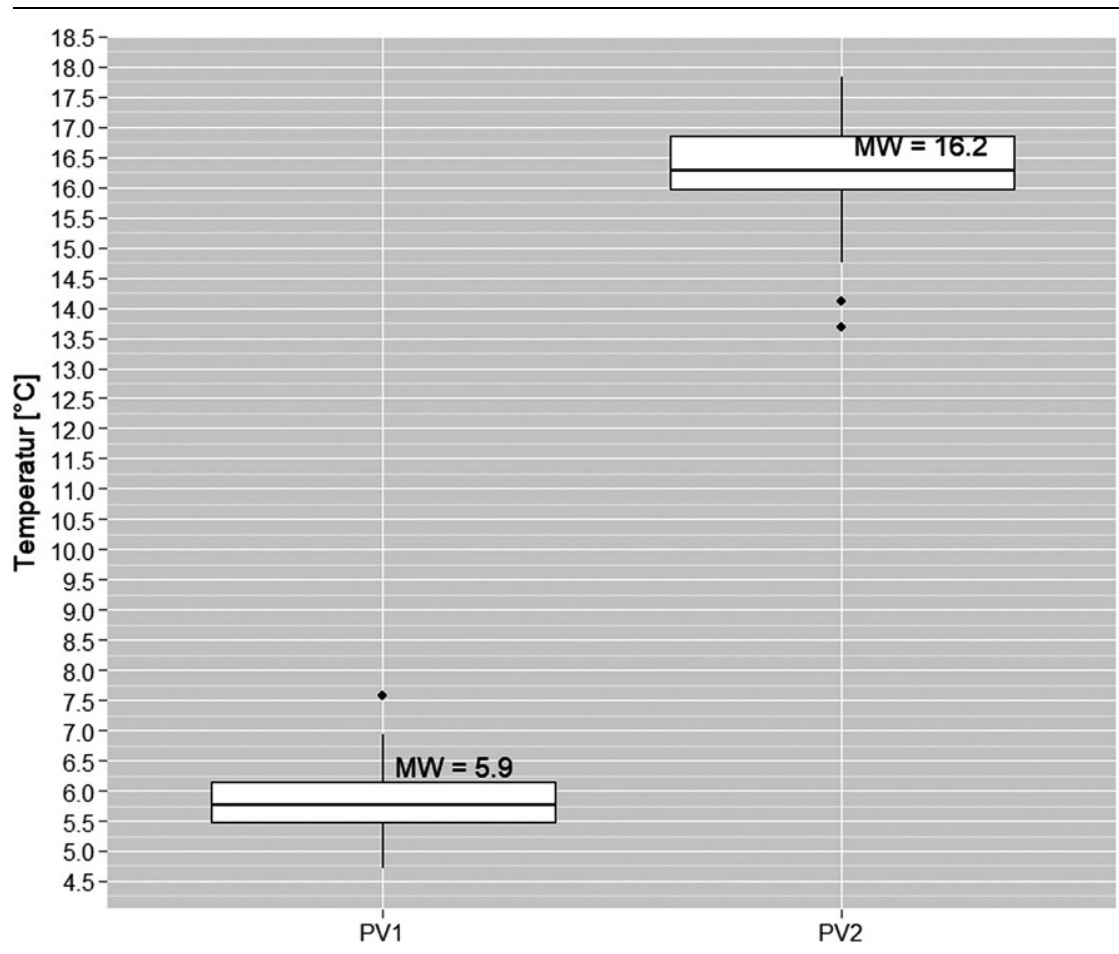

Abb. 2 Vergleich der Grundwassertemperaturen während der Pumpversuche

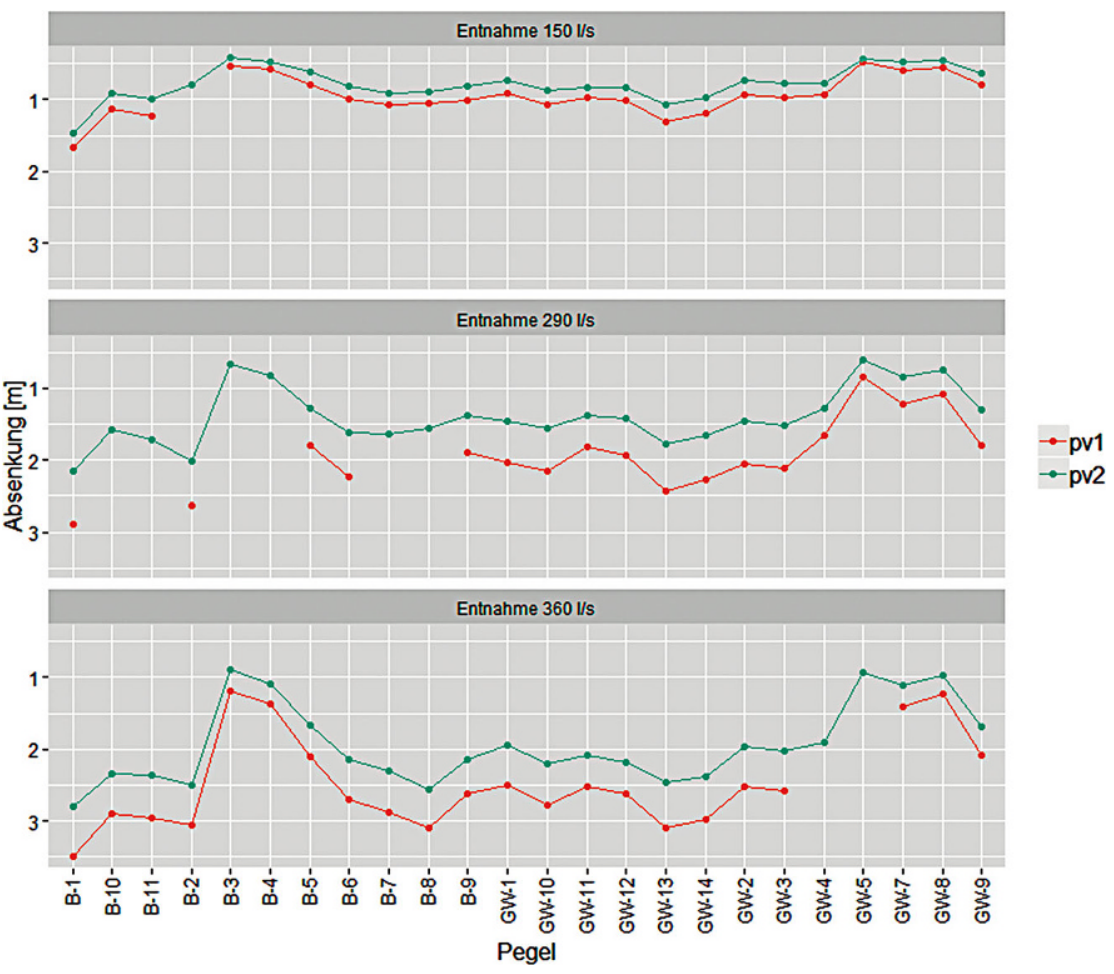

Abb. 3 Absenkung während der stationären Zustände der Pumpversuche

\section{Quantitative Beweissicherung}

Die Auswertungen zahlreicher Pumpversuche an den Brunnenfeldern Donauinsel Nord und Nußdorf haben gezeigt, dass die Kolmation der Gewäs- sersohle der Donau und die Grundwassertemperatur zwei wesentliche Einflussfaktoren auf das quantitative Grundwasserdargebot darstellen. Beispielsweise führt nach Gutknecht et al. (1998) eine Änderung der Grundwas- sertemperatur von 10 auf $20^{\circ} \mathrm{C}$ schon zu einer Erhöhung des Dargebots von $30 \%$. Durch das 100-jährliche Hochwasser im Juni 2013 wurde die Gewässersohle der Donau stark dekolmatiert. Der Kolmationsgrad nimmt nach einem Hochwasser stets ab, wobei unmittelbar nach dem Dekolmationsereignis die stärksten Änderungen zu beobachten sind (Voggenberger et al. 2012). Dies macht es sehr unwahrscheinlich, den zweiten Pumpversuch unter den gleichen Rahmenbedingungen wie beim ersten durchzuführen. Bei Berücksichtigung der natürlichen Verhältnisse würde z.B. ein kolmationsbedingter Rückgang der Infiltrationsrate - wie nach einem Hochwasser zu erwarten fälschlicherweise den Baumaßnahmen zugeschrieben werden. Die Auswirkungen der Baumaßnahmen und ein Vergleich der beiden Pumpversuche werden daher mithilfe eines gekoppelten Grundwassermodells abgeschätzt, welches es ermöglicht, die Einflüsse der Grundwassertemperatur und der Änderung der Kolmation zu eliminieren.

\subsection{Datengrundlage Beweissicherung}

Für die quantitative Beweissicherung wurden zwei Pumpversuche, je einer vor und nach den Bautätigkeiten im Schutzgebiet, durchgeführt. Abb. 1 zeigt die Wasserstände in den Grundwassersonden GW-4, GW-5und B-6 sowie den Pegel der Donau während der beiden Pumpversuche. Tab. 1 und 2 geben die Staffelung der Entnahmemengen während der Pumpversuche an.

In Abb. 2 ist der Vergleich der Grundwassertemperaturen während der beiden Pumpversuche dargestellt. Die Mittelwerte liegen im Pumpversuch 1 bei $5,9^{\circ} \mathrm{C}$ und im Pumpversuch 2 bei $16,2^{\circ} \mathrm{C}$ und geben somit einen weiten Bereich der natürlichen jährlichen Varianz wieder.

\subsection{Hydrogeologische Verhältnisse im Projektgebiet}

Das Uferfiltratdargebot ist im Wesentlichen von den Durchlässigkeiten der Donausohle und des Grundwasserleiters (Donaubegleitstrom) abhängig.

Die Viskosität („Zähigkeit“) eines Mediums hat einen nicht zu vernachlässigenden Einfluss auf die Grundwasserfließgeschwindigkeit bzw. den Durchlässigkeitsbeiwert $k_{f}$. Somit ist für die Beschreibung des Infiltrationsverhaltens von Oberflächenwasser in 
Tab. 3 Mittelwert der Absenkungen während der stationären Zustände der Pumpversuche

\begin{tabular}{|l|l|l|}
\hline Entnahmerate & Absenkung-PV1 & Absenkung-PV2 \\
\hline 150 & 0,99 & 0,81 \\
\hline 290 & 1,98 & 1,44 \\
\hline 360 & 2,48 & 1,99 \\
\hline
\end{tabular}

den Grundwasserkörper sowie für die Beschreibung des Strömungsverhaltens im Grundwasserkörper selbst die Fluidtemperatur zu berücksichtigen. Im Allgemeinen nimmt die Viskosität eines Fluids mit steigender Temperatur ab.

Die Durchlässigkeit der Donausohle ist zusätzlich Kolmationsprozessen unterworfen. Diese führen $\mathrm{zu}$ einer sukzessiven Verdichtung der Sohle in Perioden mit Normalabfluss. Durch Hochwässer, welche eine bestimmte Jährlichkeit überschreiten, wird die Kolmationsschicht wieder aufgebrochen und die Verdichtungsprozesse setzen wieder verstärkt ein. Vergleiche hier$\mathrm{zu}$ die Ausführungen von Gutknecht et al. (1998), Blaschke et al. (2003) und Saueregger (2009) zur Kolmation von Flussbetten.

Weder die Durchlässigkeit der Donausohle noch die Wassertemperatur sind im Jahresverlauf konstant. Abb. 3 zeigt die Absenkung in den beobachteten Grundwassersonden während der stationären Zustände der durchgeführten Pumpversuche. Die Absenkungen während des Pumpversuchs 2, welcher nach Herstellung des Bauwerks durchgeführt wurde, sind geringer als die Absenkungen im Pumpversuch 1. Dies ist auf die geänderten Rahmenbedingungen, die erhöhte Durchlässigkeit des Grundwasserleiters wegen höherer rung des Kolmationseffekts der Donausohle durch zwei Hochwässer der Jährlichkeit 1 zwischen den Pumpversuchen zurückzuführen. In Tab. 3 sind die Mittelwerte der Absenkungen für die einzelnen Entnahmeraten zusammengestellt.

\subsection{Untersuchungskonzept}

Um den Einfluss des Bauwerks auf den Grundwasserhaushalt darstellen zu können, müssen die Einflüsse der Kolmation und der Temperatur auf die Zielgröße der Absenkung eliminiert werden. Dazu wurde ein numerisches Grundwassermodell erstellt. Als Datengrundlage für die Kalibrierung und Validierung dienen die zwei Pumpversuche, die vor bzw. nach Errichtung des Bauwerks durchgeführt wurden.

Nach Abschn. 3.1. und 3.2. decken die Rahmenbedingungen während der beiden Pumpversuche einen wesentlichen Teil des natürlich auftretenden Spektrums ab.

Der Einfluss des Forschungsgerinnes auf den Grundwasserhaushalt wird daher anhand von Modellierungsergebnissen wie folgt untersucht: Für jeden Pumpversuch wird je ein zusätzliches Szenario modelliert, indem im PV1 das Bauwerk mitberücksichtigt und im PV2
Wassertemperatur und die Verringe- sein Einfluss entfernt wird. Dadurch kann der Einfluss des Bauwerks auf die Strömungsverhältnisse an zwei Zuständen (PV1 und PV2) anhand von je zwei Szenarien (mit und ohne Bauwerkseinfluss) untersucht werden.

\subsection{Grundwassermodell}

\subsubsection{Aufbau}

Es wurde ein dreidimensionales, instationäres numerisches Grundwassermodell mit der Modellierungssoftware Processing Modflow (Chiang und Kinzelbach 2001) aufgebaut. Abb. 4 zeigt einen Übersichtslageplan des Untersuchungsgebiets. Die räumliche Ausdehnung des Modells in Fließrichtung der Donau erstreckt sich vom unteren Ende der Schutzzone III auf Höhe der Nordbrücke (Stromkilometer 1932,60) bis zur Abzweigung des Donaukanals flussauf (Stromkilometer 1934,00). Quer zur Donau wird das Modell in Richtung 19. Bezirk durch die Dichtwand entlang des Brunnenschutzgebietes begrenzt. In Richtung der Donauinsel endet das Modell etwa $10 \mathrm{~m}$ im Ufer der Donauinsel entlang der Grundwasserbeobachtungssonden. Die Lage des Grundwasserstauers wurde aus den vorhandenen Bohrprofilen im Untersuchungsgebiet räumlich interpoliert. Die Bohrpfähle entlang des Einlaufs des Schleusenkanals wurden entsprechend der Baupläne durch inaktive Zellen im Grundwassermodell berücksichtigt.

Über das Brunnenfeld sind 13 Grundwassermessstellen und 11 Brunnen verteilt, welche mit Datenloggern zur kontinuierlichen Messung des Wasser-

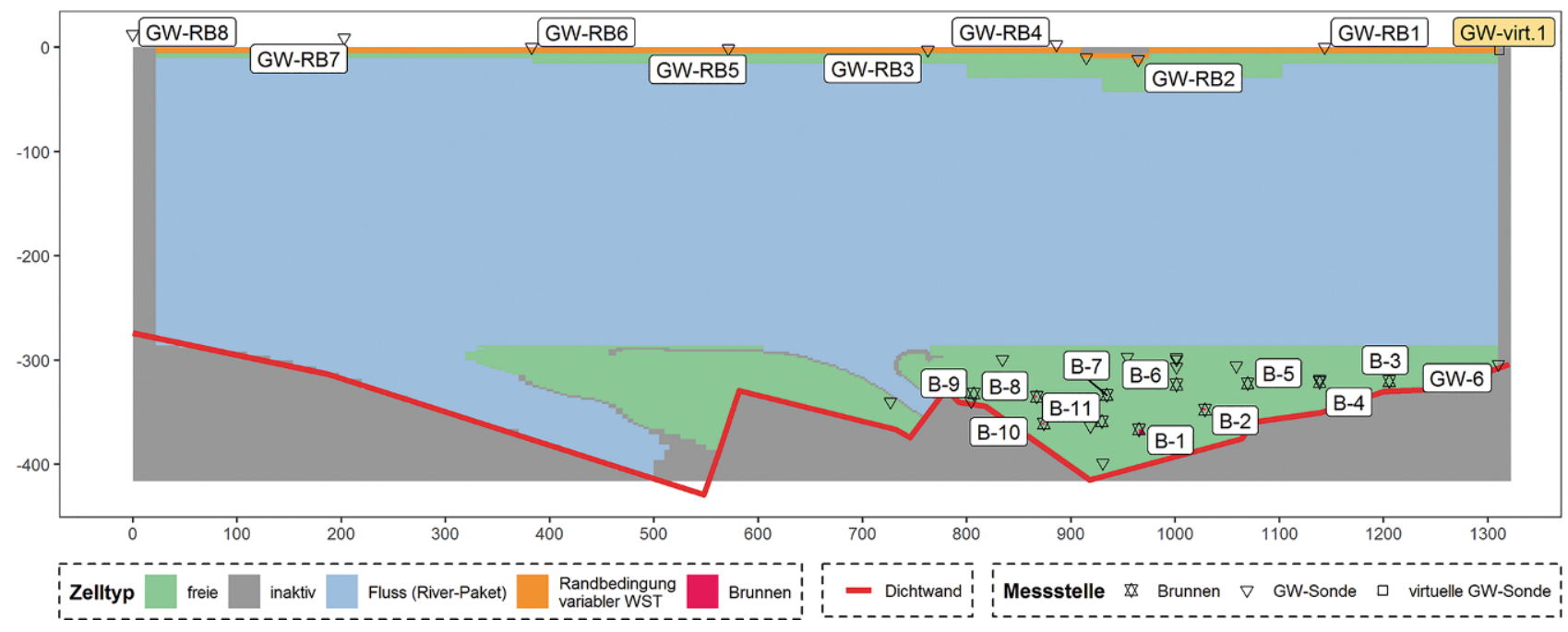

Abb. 4 Randbedingungen im Modell 
Tab. 4 Güte der Modellanpassung an den Pumpversuch 1

\begin{tabular}{|c|c|c|c|c|c|}
\hline Messstelle & $M Q A$ & NSE & Messstelle & MQA & NSE \\
\hline B-1 & 0,15 & 0,97 & GW-2 & 0,15 & 0,96 \\
\hline B-10 & 0,18 & 0,94 & GW-3 & 0,14 & 0,97 \\
\hline B-11 & 0,25 & 0,89 & GW-4 & 0,13 & 0,96 \\
\hline B-2 & 0,19 & 0,96 & GW-5 & 0,10 & 0,66 \\
\hline B-3 & 0,19 & 0,64 & GW-7 & 0,09 & 0,96 \\
\hline B-4 & 0,12 & 0,90 & GW-8 & 0,16 & 0,79 \\
\hline B-5 & 0,11 & 0,97 & GW-9 & 0,11 & 0,97 \\
\hline B-6 & 0,15 & 0,97 & GW-10 & 0,20 & 0,94 \\
\hline B-7 & 0,17 & 0,95 & GW-11 & 0,12 & 0,97 \\
\hline B-8 & 0,14 & 0,97 & GW-12 & 0,16 & 0,96 \\
\hline B-9 & 0,15 & 0,96 & GW-13 & 0,14 & 0,98 \\
\hline GW-1 & 0,16 & 0,96 & GW-14 & 0,16 & 0,97 \\
\hline
\end{tabular}

Tab. 5 Güte der Modellanpassung an den Pumpversuch 2

\begin{tabular}{|c|c|c|c|c|c|}
\hline Messstelle & MQA & NSE & Messstelle & MQA & NSE \\
\hline B-1 & 0,33 & 0,91 & GW-2 & 0,28 & 0,92 \\
\hline B-10 & 0,24 & 0,96 & GW-3 & 0,29 & 0,92 \\
\hline B-11 & 0,28 & 0,94 & GW-4 & 0,23 & 0,94 \\
\hline B-2 & 0,29 & 0,95 & GW-5 & 0,17 & 0,87 \\
\hline B-3 & 0,15 & 0,94 & GW-7 & 0,25 & 0,84 \\
\hline B-4 & 0,22 & 0,91 & GW-8 & 0,13 & 0,94 \\
\hline B-5 & 0,53 & 0,80 & GW-9 & 0,26 & 0,91 \\
\hline B-6 & 0,33 & 0,92 & GW-10 & 0,27 & 0,94 \\
\hline B-7 & 0,22 & 0,96 & GW-11 & 0,21 & 0,96 \\
\hline B-8 & 0,21 & 0,97 & GW-12 & 0,24 & 0,95 \\
\hline B-9 & 0,26 & 0,95 & GW-13 & 0,31 & 0,92 \\
\hline GW-1 & 0,27 & 0,92 & GW-14 & 0,30 & 0,93 \\
\hline
\end{tabular}

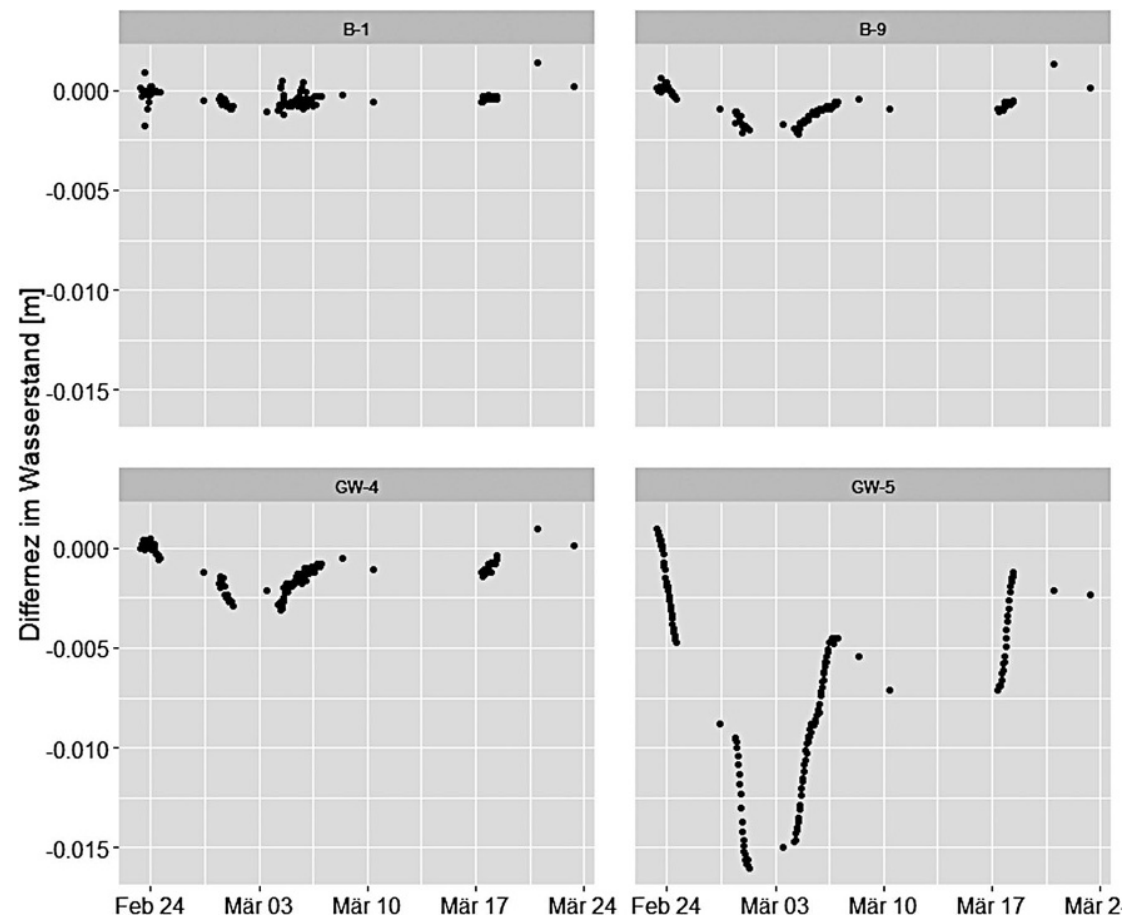

Abb. 5 Differenz im Wasserstand durch Berücksichtigung des Bauwerks im PV1 stands ausgestattet wurden. Entlang des Donauinselufers sind im Modellgebiet weitere 7 Piezometer mit Datenloggern ausgestattet und auch der Pegel der Donau wird kontinuierlich aufgezeichnet.

Es werden vier Arten von Randbedingungen entsprechend Abb. 4 angesetzt. Undurchlässige Ränder werden entlang der Dichtwand in Richtung 19. Bezirk und an den Rändern quer zur Donau angesetzt. Entlang der Donauinsel wird der Wasserstand entsprechend den Messungen in den Piezometern zeitlich variabel definiert. Der Zustrom aus der Donau in die jeweilige Zelle unterhalb des Flusses wird mit dem River-Packet der Modellierungssoftware definiert. Er ist dementsprechend von der Durchlässigkeit der Gewässersohle, dem Pegel im Fluss und dem momentanen Wasserstand in der Zelle unterhalb der Donau abhängig.

\subsubsection{Kalibrierung - Validierung}

Das Modell wurde an die Daten des Pumpversuch 1 angepasst, indem schrittweise sowohl die Ausdehnung homogener Bereiche im Grundwasserkörper wie auch deren Durchlässigkeitsbeiwerte adaptiert wurden.

Tab. 4 zeigt die Anpassung anhand der Parameter Mittlere Quadratische Abweichung (MQA) und Nash-SutcliffeKriterium (NSE). Mit einem Maximalwert des MQA von $20 \mathrm{~cm}$ und einem Mittelwert des NSE von größer 0,92 kann von einer sehr hohen Modellgüte ausgegangen werden.

Die Validierung des Modells erfolgte anhand der Daten des Pumpversuchs 2. Die Änderung der Rahmenbedingungen wurde wie folgt berücksichtigt: Die Durchlässigkeitsbeiwerte des Grundwasserkörpers wurden entsprechend den Viskositätsänderungen, hervorgerufen durch die gemittelte Temperaturänderung über das gesamte Untersuchungsgebiet, angepasst. Der Durchlässigkeitsbeiwert der Donausohle kann im vorliegenden Untersuchungsaufbau nicht direkt gemessen werden. Er wurde wiederum innerhalb des Vertrauensbereiches schrittweise so adaptiert, dass eine bestmögliche Übereinstimmung zwischen berechneten und beobachteten Wasserständen vorlag.

Aus betrieblichen Gründen war bei der Erhöhung der Entnahmerate von 360 auf 700l/s eine kurzfristige Abschaltung der Pumpen notwendig. Der zeitliche Verlauf der Entnahmeraten für diesen kurzen Zeitraum ist nicht 
Hier steht eine Anzeige.

Springer 
Hier steht eine Anzeige.

Springer 


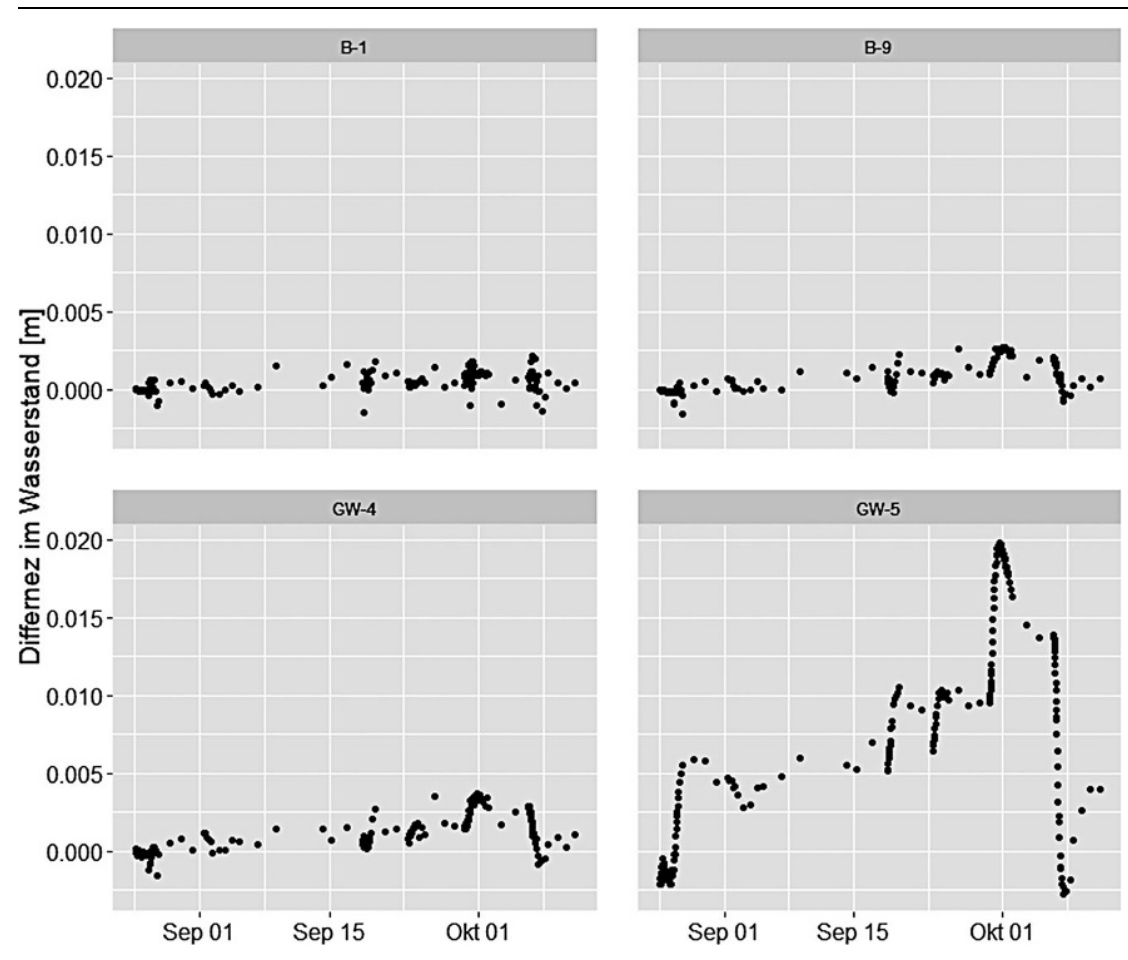

Abb. 6 Differenz im Wasserstand durch Elimination des Einflusses des Bauwerks im PV2

exakt bekannt. Aus diesem Grund wurde dieser kurze Zeitraum nicht bei der Berechnung der Güteparameter des Modells berücksichtigt.

Tab. 5 zeigt die Anpassung anhand der Güteparameter. Der Mittelwert des MQA von $26 \mathrm{~cm}$ und Mittelwert des NSE von 0,92 bestätigen die gute Modellanpassung.

\subsection{Untersuchungsergebnisse}

Die Errichtung des Forschungsgerinnes führt im Wesentlichen zu einer Verringerung des Grundwasserströmungsquerschnitts des donaubegleitenden Grundwasserleiters. Im numerischen Modell wird das Bauwerk durch Zellen repräsentiert. Durch die Inaktivierung dieser Zellen wird der Einfluss des Bauwerks auf den Grundwasserhaushalt in das Modell übertragen.
Wie bereits beschrieben, werden für beide Pumpversuche Szenarien berechnet, indem diese Querschnittsminderung im PV1 berücksichtigt wird bzw. im PV2 jene Zellen aktiviert werden, die das Forschungsgerinne repräsentieren. Durch den Vergleich der Wasserstände und Zuflussraten zwischen den Szenarien kann der Einfluss des Bauwerks auf den Grundwasserleiter abgeschätzt werden.

\subsubsection{Einfluss auf den Wasserstand}

Stellt man die Veränderungen des Wasserstands in ausgewählten Sonden zwischen den Szenarien ohne und mit Berücksichtigung der Querschnittsänderung durch das Forschungsgerinne in den Pumpversuchen dar, erhält man die Unterschiede für den ersten (Abb. 5) und zweiten Pumpversuch (Abb. 6). Die errechneten Werte liegen mit maximal $2 \mathrm{~cm}$ in der Sonde GW-5 bei einer Entnahme von 7001/s unter der Genauigkeit der Modellanpassung.

\subsubsection{Einfluss auf die Zustromrate}

Für die einzelnen Szenarien mit und ohne Bauwerk wurden jeweils die $\mathrm{Zu}$ stromraten aus Zone 1 in die Zone 2 berechnet. Abb. 7 zeigt die definierten Zonen für die Berechnung der Zustromrate. Dabei bezeichnet Zone 1 den Bereich zwischen Donaukanaleinlauf und Bauwerk, Zone 2 die Zone zwischen Bauwerk und Brunnenfeld. Zur Beurteil des Bauwerkseinflusses auf die Zustromraten wurden jeweils Differenzen für jeden einzelnen Pumpversuch zwischen der Situation mit und der

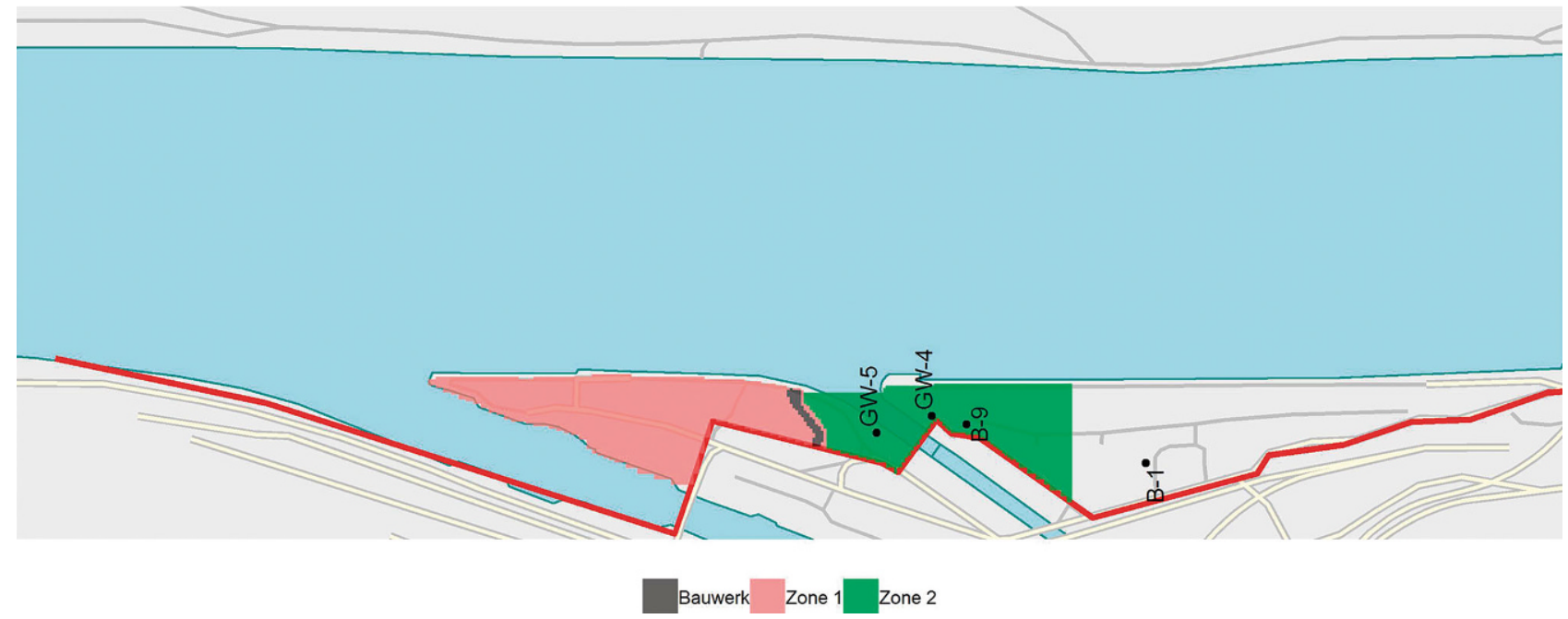

Abb. 7 Zonen der Zustrombudgetierung 
Tab. 6 Differenz der Zustromrate aus Zone 1 in Zone 2 zwischen den Szenarien mit/ohne Bauwerk in l/s

\begin{tabular}{|l|l|l|}
\hline Entnahmerate & PV1 & PV2 \\
\hline 150 & 0,4 & 0,4 \\
\hline 290 & 0,5 & 1,6 \\
\hline 360 & 0,6 & 0,7 \\
\hline 700 & - & 1,1 \\
\hline
\end{tabular}

Situation ohne Bauwerk gebildet. Die Differenzen ergeben somit den Einfluss des Bauwerks auf die beiden Pumpversuche. Die Differenz der Zustromrate aus Zone 1 in Zone 2 der beiden Szenarien (mit Bauwerk, ohne Bauwerk) ist für die beiden Pumpversuche in Tab. 6 dargestellt.

Die berechnete, maximale Änderung der Zustromrate durch die Berücksichtigung des Bauwerks beträgt 1,11/s bei einer Entnahmerate von 7001/s aus dem Brunnenfeld während des Pumpversuchs 2. Die Unsicherheiten in der Anpassung des Modells an die Beob- achtungen während der Kalibrierung liegen somit weit höher als die berechneten Unterschiede zwischen den Szenarien.

\section{Schlussfolgerungen}

Die berechneten Unterschiede zwischen den Szenarien mit und ohne Berücksichtigung der Querschnittsminderung durch das Bauwerk für die beiden Pumpversuche liegen aufgrund ihrer geringen Ausprägung sowohl beim Wasserstand wie auch bei der Zustromrate unter der Genauigkeit der Modellanpassung an die Beobachtungen.

Es ist somit nicht von einer Beeinflussung der quantitativen Grundwasserverhältnisse des Brunnenfelds Nußdorf durch das Forschungsgerinne auszugehen.

Förderung Die Bearbeitungen wurden im Rahmen des Projekts SEDDON (Se- dimentforschung und -management an der Donau) durchgeführt, welches aus dem ETZ-Programm AT-HU 2007-2013 mit EU-Mitteln (EFRE) kofinanziert wurde.

Funding Open access funding provided by University of Natural Resources and Life Sciences Vienna (BOKU).

Open Access Dieser Artikel wird unter der Creative Commons Namensnennung 4.0 International Lizenz (http:// creativecommons.org/licenses/by/4. $0 /$ deed.de) veröffentlicht, welche die Nutzung, Vervielfältigung, Bearbeitung, Verbreitung und Wiedergabe in jeglichem Medium und Format erlaubt, sofern Sie den/die ursprünglichen Autor(en) und die Quelle ordnungsgemäß nennen, einen Link zur Creative Commons Lizenz beifügen und angeben, ob Änderungen vorgenommen wurden.

\section{Literatur}

Blaschke, A. P., Steiner, K.-H., Schmalfuss, R. Gutknecht, D. Sengschmitt, D. (2003): Clogging Processes in Hyporheic Interstices of an Impounded River, the Danube at Vienna, Austria; International Review of Hydrobiology, vol. 88 3-4, p. 397-413

Chiang, W. H. and Kinzelbach, W. (2001): 3DGroundwater Modeling with PMWIN. First Edition. Springer Berlin Heidelberg New York. ISBN 3-540 67744-5, $346 \mathrm{pp}$
Gutknecht, D, Blaschke, A, D, Schmalfuss, R., Sengschmitt, D., Steiner, K.-H., Reichel, K G., Feregyhazy, H., Herndl, G. H., Battin, T. J. Dreher, J. E. (1998): Kolmationsprozesse am Beispiel des Stauraums Freudenau, Schriftenreihe der Forschung im Verbund, Band 44

Saueregger, G. (2009): Untersuchung von Kolmationsprozessen in der freien Fließstrecke der Lutz mittels eines physikalischen Modells. Masterarbeit, TU Graz.
Voggenberger, C., Wögerer, C., Biedrawa, C. Prantl, M. (2012): Brunnenfelder Donauinsel Nord und Nussdorf Pumpversuch 2011, GRUPPE WASSER, Bericht im Auftrag der Stadt Wien MA 31

Hinweis des Verlags Der Verlag bleibt in Hinblick auf geografische Zuordnungen und Gebietsbezeichnungen in veröffentlichten Karten und Institutsadressen neutral. 